\title{
ON THE APPLICATION OF DYNAMIC PROGRAMMING TO A CLASS OF IMPLICIT VARIATIONAL PROBLEMS*
}

\author{
BY \\ RICHARD BELLMAN (The Rand Corporation) \\ AND \\ JOHN M. RICHARDSON (Hughes Aircraft Company)
}

1. Introduction. A large and important class of variational problems has the following form. Given a vector equation of the form

$$
\frac{d x}{d t}=g(x, y), \quad x(0)=c,
$$

where $x$ is an $N$-dimensional vector, we wish to determine an $m$-dimensional vector $y$ so as to minimize a given criterion functional

$$
J(y)=\int_{0}^{T} h(x, y) d t
$$

where $h(x, y)$ is a given scalar function.

The vector $y$ may be subject to constraints of the form

$$
r_{i}(x, y) \leq 0, \quad i=1,2, \cdots, q .
$$

In problems involving "terminal control," we meet the problem of minimizing a function only of the final state

$$
I(y)=k[x(T)] .
$$

A problem of this nature occurs when we wish to have the system in some specified state $x_{0}(T)$ at time $T$, without caring how the system gets there. This is usually an idealization, in the sense that a more realistic problem will involve a combination of a criterion of the type appearing in (2) together with some measure of the value of the final state.

As has been shown in some recent publications, see [1], where further references may be found, a variety of problems of this nature arising in economic and engineering control processes may be solved computationally by combining the theory of dynamic programming with modern digital computers.

In recent years, problems of less explicit nature have become more frequent. Thus, for example, what is called the "bang-bang" control problem requires that $y$ be chosen so that the system tend to a specified equilibrium state as rapidly as possible; see [2].

The upper limit of integration is thus not predetermined, but is rather a function of the choice of the vector $y$. In place of a formulation in precise analytic terms of the type appearing in (2) or (4), we encounter an implicit criterion of the following type:

"When $x$ satisfies a set of conditions $C_{1}, C_{2}, \cdots, C_{p}$ for the first time, we want a given scalar function of $x$ to be as small as possible."

*Received June 9, 1958. 
A particular example of a problem of this nature, equivalent to one we shall discuss in more detail below, is one in which we require that a preassigned function be a minimum for the first value of $T$ for which $x_{1}(T)=a_{1}$, a given value.

A number of quite interesting existence and uniqueness questions arise in conjunction with problem statements of the foregoing kind. These will be discussed at some time in the future. Here we are interested in describing a technique which can be used to obtain computational solutions via the functional equation path of dynamic programming.

The problem becomes of even more interesting nature if we insert some stochastic influences into the process. Let the governing equation be

$$
\frac{d x}{d t}=g(x, y, r), \quad x(0)=c,
$$

where $r$ is a random vector. We now wish to minimize an expected deviation, or say the probability that the deviation exceeds a given critical value.

Once again, let us point out that the rigorous groundwork for these questions remains to be laid. However, as we shall see below, we have a simple method for postponing this type of investigation.

As is to be expected, certain simplifications are possible if the underlying equations are linear, i.e. of the form

$$
x_{n+1}=A x_{n}+y_{n}+r_{n}, \quad x_{0}=c,
$$

and the criteria quadratic. We shall discuss these cases in some detail since they are of some importance in connection with the application of the method of successive approximations.

Throughout, our aim will be to illustrate the applicability of the functional equation technique of dynamic programming to the computational solution of questions of this kind which appear in many ways to be outside the domain of the classical calculus of variations.

2. Preliminaries. Since, as mentioned above, we are primarily interested in a computational solution of implicit variational problems of the type described in the foregoing section, we shall pose our problem in discrete terms. The recurrence relations we derive will then be ready for use in a digital computer.

In place of the differential relation of (1.4), consider the difference equation

$$
x_{n+1}=g\left(x_{n}, y_{n}, r_{n}\right), \quad x_{0}=c, \quad n=0,1, \cdots, N .
$$

One of the advantages of formulating problems in this fashion is that there are now no conceptual difficulties concerning the meaning of random functions or the existence of minimizing functions. In return, sometime or other we must show that the limit of the discrete process exists, and, preferably, yields the continuous process. For a start in this direction, see [3].

In order to illustrate the method in simple fashion, we shall consider a two-dimensional process,

$$
\begin{aligned}
& x_{1}(n+1)=x_{1}(n)-y_{1}(n)-r_{1}(n), \quad x_{1}(0)=c_{1}, \\
& x_{2}(n+1)=g_{2}\left[x_{1}(n), x_{2}(n), y_{2}(n), r_{2}(n)\right], \quad x_{2}(0)=c_{2} .
\end{aligned}
$$


The aim of the process is to choose $y_{1}(n)$ and $y_{2}(n)$, subject to constraints of the form

$$
0<a_{1} \leq y_{1}(n) \leq a_{2}, \quad 0 \leq b_{1} \leq y_{2}(n) \leq b_{2}
$$

so as to minimize the expected value of $\left[x_{2}(m)-x_{0}\right]^{2}$, where $m$ is the "time" at which $x_{1}(m)=0$. The $r_{i}(n)$ are independent random variables with given distributions.

The expected value is over the random variables $r_{1}$ and $r_{2}$, where $r_{1}$ can depend upon the choice of $y_{1}$ and $y_{2}$, but, in any case is subject to the condition that

$$
y_{1}(n)+r_{1}(n) \geq a_{3}>0 .
$$

It follows that $x_{1}(n)$ is steadily decreasing as $n$ increases.

The recurrence relation in (1) is valid until $x_{1}(n)=0$. Properly, we should write

$$
x_{1}(n+1)=\max \left[0, x_{1}(n)-y_{1}(n)-r_{1}(n)\right] .
$$

The process ends as soon as $x_{1}$ assumes the value zero.

3. Functional equations. It is clear that the minimum of the expected value of $\left[x_{2}(n)-x_{0}\right]^{2}$ depends upon $c_{1}$ and $c_{2}$ and only upon these variables assuming all other functions and distributions known and fixed. Let us then write

$$
f\left(c_{1}, c_{2}\right)=\min _{y_{i}} \exp _{r}\left[x_{2}(n)-x_{0}\right]^{2} .
$$

We have

$$
f\left(0, c_{2}\right)=\left(c_{2}-x_{0}\right)^{2},
$$

and the principle of optimality, see [1], yields the functional equation

$$
f\left(c_{1}, c_{2}\right)=\min _{y_{1}, y_{2}}\left[\exp _{r_{1}, r_{2}} f\left(c_{1}-y_{1}-r_{1}, g\left(c_{1}, c_{2}, y_{2}, r_{2}\right)\right]\right. \text {. }
$$

There is no difficulty in treating the case in which the distribution of random effects depends upon the decisions that are made.

4. Probability of deviation. In place of mean-square deviation, let us consider the problem of determining $y_{1}$ and $y_{2}$ so as to minimize the probability that $\left|x_{2}-x_{0}\right| \geq d$.

As above, let

$$
f\left(c_{1}, c_{2}\right)=\min _{y_{i}} \text { prob }\left[\left|x_{2}-x_{0}\right| \geq d\right]
$$

Then

$$
\begin{aligned}
f\left(0, c_{2}\right) & =1, & & \left|c_{2}-x_{0}\right| \geq d, \\
& =0, & & \left|c_{2}-x_{0}\right|<d .
\end{aligned}
$$

while $f\left(c_{1}, c_{2}\right)$ satisfies the same functional equation as in (3.3).

5. Discussion of computational solution. In order to determine the function $f\left(c_{1}, c_{2}\right)$ using a digital computer, we employ a discrete grid in $\left(c_{1}, c_{2}\right)$-space. Let $c_{1}$ assume only the sequence of values $0, \delta, 2 \delta, \cdots$, and $c_{2}$ a sequence of values $0, \Delta, 2 \Delta, \cdots$. Since $c_{1}$ is monotonically decreasing as the process continues, we can use it as a "time" variable. Write

$$
f\left(k \delta, c_{2}\right) \equiv f_{k}\left(c_{2}\right) .
$$

Then (3.3) may be written 


$$
f_{k}\left(c_{2}\right)=\min _{y_{1}, y_{2}}\left[\exp _{r_{1}, r_{2}} f_{p}\left[g\left(k \delta, c_{2}, y_{2}, r_{2}\right)\right]\right],
$$

where $p$ is determined by the condition

$$
p=\left[\left(c_{1}-y_{1}-r_{1}\right) / \delta\right],
$$

the greatest integer contained in $\left(c_{1}-y_{1}-r_{1}\right) / \delta$.

Since $g\left(k \delta, c_{2}, y_{2}, r_{2}\right)$ in general will not be an integral multiple of $\Delta$, we can either take as its value the nearest integer multiple of $\Delta$, as we did in (3), or we can use interpolation, if more accurate results are desired.

The value of $f_{0}\left(c_{2}\right)$ is determined by the relation

$$
f_{0}\left(c_{2}\right)=\left(c_{2}-x_{0}\right)^{2} .
$$

Consequently (2) furnishes a recurrence relation which enables us to compute the function $f_{k}\left(c_{2}\right)$ in terms of $f_{n}\left(c_{2}\right)$ for $n=0,1, \cdots, k-1$. We thus have a feasible computational scheme.

6. Deterministic process. Returning to a purely deterministic process, as specified by (1.1), we may wish to determine $y$ so that $x$ is in some desired state at some subsequent time. One way of attacking this problem is to treat the problem of minimizing $\left[x_{2}(T)-x_{0}\right]^{2}$, where $T$ is the first time at which $x_{1}(T)$ has its desired value. The functional equations are as above, without the averaging over the random behavior.

7. Linear equations and quadratic criteria. In general, the application of a straightforward functional equation approach is limited by dimensionality difficulties in the sense that functions of three or more variables cannot be readily stored in a fast memory. Consequently, the techniques described above must be aided and abetted by successive approximations of various types, a subject which has been discussed elsewhere. If, however, the guiding equations are linear, and the criteria function quadratic, then the sequence of functions $\left\{f_{n}(c)\right\}$ will consist of a sequence of quadratic functions in $c$. These functions are determined once the coefficients are determined. As we shall see, reasonably simple recurrence relations exist connecting the coefficients of $f_{n}(c)$ with those of $f_{n-1}(c)$.

Consider, to begin with, the problem of choosing the $y_{i}$ so as to minimize the expected mean-square deviation

$$
J_{T}(y)=\exp _{r}\left[(x(T)-a, x(T)-a)+\sum_{k=0}^{T}\left(y_{k}, B y_{k}\right)\right] .
$$

Here $T$ assumes the values $0,1,2, \cdots, B$ is a positive definite matrix, $a$ is a specified state vector, $x$ and $y$ are related by means of the linear relations

$$
x_{n+1}=A x_{n}+y_{n}+r_{n}, \quad x_{0}=c,
$$

where $\left\{r_{i}\right\}$ is a set of independent, random vectors with identical distributions.

The process is assumed to proceed in the following fashion. We observe $c$, the initial state, and on this basis and the foregoing information, choose $y_{0}$, the initial control vector. Then a random effect $r_{0}$ occurs, yielding by way of (2) a new state vector $A c+y_{0}+r_{0}$. The process then continues in this way, stage-by-stage, a "feedback control" process.

Although this problem can be, and has been, treated by straightforward variational techniques, we shall treat it by functional equation methods. There is some merit in 
doing this even in this case, and in addition we shall prepare the way for the following section devoted to a process of random duration.

Define the new sequence of functions $\left\{f_{T}(c)\right\}$ by means of the relation

$$
f_{T}(c)=\min _{|\nu|} J_{T}(y) \text {. }
$$

Then

$$
f_{0}(c)=(c-a, c-a),
$$

and the principle of optimality yields the recurrence relation

$$
f_{n}(c)=\min _{\nu_{0}} \exp _{r_{0}}\left[\left(y_{0}, B y_{0}\right)+f_{n-1}\left(A c+y_{0}+r_{0}\right)\right]
$$

for $n=1,2, \cdots$.

Let us now show inductively that each $f_{n}(c)$ may be written in the form

$$
f_{n}(c)=\left(c, M_{n} c\right)+2\left(b_{n}, c\right)+u_{n} .
$$

The result is obviously so for $n=0$.

Substituting in (5), we have

$$
\begin{aligned}
f_{n}(c)=\min _{\nu_{0}} \exp _{r_{0}}\left[\left(y_{0}, B y_{0}\right)\right. & +\left[A c+y_{0}+r_{0}, M_{n-1}\left(A c+y_{0}+r_{0}\right)\right] \\
& \left.+2\left(b_{n-1}, A c+y_{0}+r_{0}\right)+u_{n-1}\right] .
\end{aligned}
$$

Taking expected values and using the result that

$$
\min _{\nu}[(y, C y)+2(g, y)]=-\left(g, C^{-1} g\right),
$$

whenever $C$ is positive definite, we see that $f_{n}(c)$ has the form stated in (6). Carrying through the calculations, we obtain recurrence relations connecting $M_{n}, b_{n}$ and $d_{n}$ with $M_{n-1}, b_{n-1}$ and $d_{n-1}$.

8. Linear process of random duration. Consider now a system specified by the equations

$$
\begin{aligned}
& u_{n+1}=u_{n}-r_{1 n}, \quad u_{0}=c_{0}, \\
& x_{n+1}=A x_{n}+y_{n}+r_{n}, \quad x_{0}=c,
\end{aligned}
$$

where $u_{n}$ and $r_{1 n}$ are scalars, $x_{n}, y_{n}$ and $r_{n}$ vectors. The process ends whenever $u_{n}$ becomes zero or negative.

The quantity $r_{1 n}$ is a uniformly positive random variable, so that the process is always finite. The control vectors $y_{n}$ are to be chosen so as to minimize the expected value of

$$
J(y)=[x(m)-a, x(m)-a]+\sum_{k=0}^{m}\left(y_{k}, B y_{k}\right),
$$

where $m$ is itself a random variable determined by the condition that it is the first integer for which $u_{m}$ is negative or zero.

Write

$$
f\left(c_{0}, c\right)=\min _{\nu} \exp _{r} J(y)
$$


Then

$$
f(0, c)=(c-a, c-a)
$$

and

$$
f\left(c_{0}, c\right)=\min _{\nu_{0}} \exp _{r_{0}} f\left(c_{0}-r_{10}, A c+y_{0}+r_{0}\right) .
$$

Assume, as previously, that $c_{0}$ can assume only a discrete set of values with a similar condition on $r_{10}$. Let, suitably normalized, $c_{0}$ take the values $0,1, \cdots$, and $r_{10}$ only the range of values $d_{1}, d_{1}+1, \cdots, d_{2}$. Then, writing

$$
f(k, c) \equiv f_{k}(c), \quad k=0,1,2, \cdots,
$$

we may write (5) in the form

$$
f_{n}(c)=\min _{\nu_{0}} \exp _{r_{0}}\left\{\sum_{i=d_{1}}^{d_{2}} p_{i} f_{n-i}\left(A c+y_{0}+r_{0}\right)\right\},
$$

where

$$
p_{i}=\text { the probability that } r_{10}=i \text {. }
$$

The function $f_{k}(c)$ is identically zero for $k \leq 0$.

Once again, it is easy to see that each element of the sequence $\left\{f_{k}(c)\right\}$ is a quadratic function of $c$, of the form

$$
f_{k}(c)=\left(c, M_{k} c\right)+2\left(b_{k}, c\right)+u_{k} .
$$

The recurrence relations connecting $M_{k}, b_{k}, u_{k}$ with $M_{k-1}, b_{k-1}, u_{k-1}$ can be obtained from (7) in the way indicated above.

\section{BIBLIOGRAPHY}

1. R. Bellman, Dynamic programming, Princeton University Press, Princeton, N. J., 1957

2. R. Bellman, I. Glicksberg and O. Gross, On the "bang-bang" control problem, Quart. Appl. Math. 14, 11-18 (1956)

3. R. Bellman, Functional equations in the theory of dynamic programming-VI: A direct convergence proof, Ann. Math. 65, 215-223 (1957) 